\title{
PET/MR Imaging: A Critical Appraisal
}

\author{
Wolfgang A. Weber \\ Molecular Imaging and Therapy Service, Memorial Sloan Kettering Cancer Center, New York, New York
}

Despite considerable excitement about the potential of PET/MR imaging for the detection, staging, and functional characterization of cancer, this new technology is evolving significantly more slowly than PET/CT. This slower evolution is due partly to ongoing technologic challenges (e.g., accurate attenuation correction of PET images) but also to the complex logistics of combining a whole-body PET scan with whole-body or organ-specific MR imaging. Most PET/MR imaging research published so far has focused on cancer staging and restaging in patients undergoing ${ }^{18} \mathrm{~F}-\mathrm{FDG} \mathrm{PET} / \mathrm{CT}$ as the standard of care. These studies have demonstrated the feasibility of clinical ${ }^{18} \mathrm{~F}-\mathrm{FDG}$ PET/MR imaging but so far have not shown substantial improvements in staging. This situation may not be unexpected in view of the fact that MR imaging has not replaced CT for staging of the malignancies for which ${ }^{18} \mathrm{~F}-\mathrm{FDG}$ PET/CT is most commonly used. Given the widespread concerns about rising health care costs in general and the costs of advanced imaging techniques in particular, establishing ${ }^{18} \mathrm{~F}-\mathrm{FDG}$ PET/MR imaging for whole-body cancer staging may be challenging because it requires more expensive equipment and longer acquisition times than ${ }^{18} \mathrm{~F}-\mathrm{FDG}$ PET/CT. An alternative approach to developing clinical PET/MR imaging is to study how stand-alone, organ-specific MR imaging can be improved by PET/MR imaging. Unfortunately, however, ${ }^{18} \mathrm{~F}$-FDG PET has significant limitations for the tumors that are most commonly studied with MR imaging (brain, liver, pancreatic, and prostate tumors). However, this situation may change with the development of new radiopharmaceuticals, such as prostate-specific membrane or gastrin-releasing peptide receptor ligands for the imaging of prostate cancer. In conclusion, PET/MR imaging has many potential advantages over PET/CT (lower radiation exposure, higher soft-tissue contrast, and multiparametric imaging). Realizing this potential in clinics likely will require new radiopharmaceuticals and applications other than whole-body cancer staging.

Key Words: physics and instrumentation; PET/MRI; PET/CT; PET; $\mathrm{MRl}$; CT

J Nucl Med 2014; 55:56S-58S

DOI: 10.2967/jnumed.113.129270

I

January 2004, The Journal of Nuclear Medicine published a supplement titled "PET/CT: Imaging Function and Structure." At that time, the first article on an integrated PET/CT system had been published less than 4 y earlier (1); nevertheless, several articles in that supplement clearly stated that integrated PET/CT will replace PET for cancer staging $(2,3)$. In the following year, more than $500 \mathrm{PET} / \mathrm{CT}$ systems were sold, and more than 1.3 million

Received Apr. 21, 2014; accepted Apr. 21, 2014.

For correspondence or reprints contact: Wolfgang A. Weber, Molecular Imaging and Therapy Service, Memorial Sloan Kettering Cancer Center, 1275 York Ave., New York, NY 10065.

E-mail: ww_petmr@outlook.com

Published online May 12, 2014.

COPYRIGHT (C 2014 by the Society of Nuclear Medicine and Molecular Imaging, Inc. whole-body PET/CT or PET studies were performed in the United States (4). Another supplement of The Journal of Nuclear Medicine, published in January 2007, focused on the impact of PET/CT on cancer management. In that supplement, Czernin et al. (5) reviewed the results of 29 studies enrolling a total of 1,841 patients. They concluded that improvements in the staging and restaging accuracies of PET/CT over PET or CT alone had been established (5).

This supplement appears at about the same time in the development of clinical MR imaging as the 2 cited supplements did in the development of clinical PET/CT: the first integrated PET/MR imaging study of the human brain was published in 2008 (6), and the first commercial whole-body PET/MR imaging systems were installed in 2010 (7). Although PET/MR imaging is still in the early stages of clinical development, it is clear that the clinical adoption of PET/MR imaging is slower than that of PET/CT. Key applications of PET/MR imaging that provide information that is clinically relevant and fundamentally different from that provided by PET/CT still need to be defined (8). The feasibility of clinical PET/MR imaging has been demonstrated in a significant number of studies (9), but technologic and logistic challenges, such as errors in attenuation correction and long scan duration, continue to be a major focus of the PET/MR imaging literature (8). Therefore, the goals of this review are to provide a brief overview of potential PET/MR imaging applications and to summarize the challenges in demonstrating the clinical benefit of PET/MR imaging.

\section{DIFFERENT CHARACTERISTICS OF PET/MR IMAGING AND PET/CT}

When PET/CT was introduced in 2000, it combined 2 robust whole-body imaging modalities. Many studies published in the 1990s had demonstrated that whole-body PET with the glucose analog ${ }^{18}$ F-FDG improved the accuracy of cancer staging when added to conventional staging (which, in most cases, was based on CT). An improvement in staging accuracy was demonstrated most extensively for lung cancer but also for recurrent colorectal cancer, squamous cell carcinomas of the head and neck, melanoma, esophageal cancer, and high-grade malignant lymphomas. For malignant lymphomas, many studies also demonstrated that ${ }^{18} \mathrm{~F}-\mathrm{FDG}$ PET was more accurate than CT alone for assessing the tumor response to chemotherapy and for predicting progression-free survival.

${ }^{18} \mathrm{~F}-\mathrm{FDG}$ PET was not intended to replace CT because CT was still needed to determine the size of tumors, to assess the infiltration of organs, and to plan surgery or radiotherapy. Importantly, CT added fundamentally new anatomic and morphologic information to PET. Conversely, the metabolic information from ${ }^{18} \mathrm{~F}-\mathrm{FDG}$ PET scans was fundamentally new to $\mathrm{CT}$ and greatly improved the detection of bone marrow metastases and the differentiation of benign from malignant adenopathy. Therefore, PET/CT was welcomed by patients, clinicians, radiologists, and nuclear medicine physicians.

The situation is fundamentally different when PET/CT is compared with PET/MR imaging. The CT part of a PET/CT study 
already provides detailed anatomic and morphologic information for cancer staging, especially if the CT scan is performed with oral and intravenous contrast material. Therefore, the step from ${ }^{18} \mathrm{~F}$ FDG PET/CT to ${ }^{18}$ F-FDG PET/MR imaging is much more incremental than the step from ${ }^{18} \mathrm{~F}$-FDG PET (or CT) to ${ }^{18} \mathrm{~F}-\mathrm{FDG}$ $\mathrm{PET} / \mathrm{CT}$. In some cases, such as lung cancer, the morphologic information from CT is actually superior to that from MR imaging. For other areas of the body, such as the neck, there is an ongoing discussion about the advantages and disadvantages of CT and MR imaging for cancer staging $(10,11)$. Therefore, it may be arguable whether the morphologic information from MR imaging can replace that from CT or whether the additional information from MR imaging justifies the substantially higher costs of ${ }^{18}$ F-FDG PET/MR imaging than of ${ }^{18} \mathrm{~F}-\mathrm{FDG} \mathrm{PET} / \mathrm{CT}$.

Some methodologic challenges add to the complexity of a comparison of ${ }^{18} \mathrm{~F}-\mathrm{FDG}$ PET/CT with ${ }^{18} \mathrm{~F}-\mathrm{FDG}$ PET/MR imaging. Multidetector $\mathrm{CT}$ is a robust technique with fairly standardized protocols and no major differences in image acquisition and reconstruction among CT systems from different vendors. In contrast, the ability to acquire multiparametric images continues to be both a major strength and a weakness of MR imaging. For clinical trials, defining a "CT protocol" that can be reproduced at many institutions is rather straightforward. In contrast, MR imaging acquisition protocols continue to be vendor-specific, and the number and type of MR imaging sequences acquired are still dependent on the preferences of individual centers. Therefore, it can be challenging to define a standard ${ }^{18} \mathrm{~F}$-FDG PET/MR imaging protocol that can be compared with ${ }^{18}$ F-FDG PET/CT in a specific clinical setting.

\section{LIMITED NUMBER OF OVERLAPPING CLINICAL INDICATIONS FOR ${ }^{18}$ F-FDG PET AND MR IMAGING}

In most of the studies on PET/MR imaging systems, research ${ }^{18} \mathrm{~F}$-FDG PET/MR imaging was performed after ${ }^{18} \mathrm{~F}$-FDG PET/CT was performed as part of the standard of care. This approach simplified patient recruitment and eliminated regulatory hurdles because patients were not exposed to additional ionizing radiation. Therefore, studies with this design are helpful for establishing the feasibility of clinical ${ }^{18} \mathrm{~F}$-FDG PET/MR imaging, but they may not necessarily identify the most promising clinical applications of PET/MR imaging technology: CT and MR imaging each has specific strengths and limitations, and ${ }^{18} \mathrm{~F}-\mathrm{FDG}$ PET/CT is more commonly used for applications in which CT is considered to provide at least the same diagnostic information as MR imaging. Therefore, focusing future studies on areas in which PET can improve the diagnostic accuracy of MR imaging may be more appropriate than studying whether wholebody ${ }^{18} \mathrm{~F}$-FDG PET/MR imaging is superior to ${ }^{18} \mathrm{~F}$-FDG PET/CT.

Unfortunately, however, ${ }^{18}$ F-FDG PET has limitations for many of the malignancies that are commonly studied with MR imaging. The imaging of primary and metastatic brain tumors is probably one of the most well-established clinical applications of MR imaging in oncology, but ${ }^{18}$ F-FDG PET studies of brain tumors are severely limited by the high rate of glucose metabolism in normal gray matter (12). Therefore, it appears unlikely that ${ }^{18} \mathrm{~F}-\mathrm{FDG}$ PET/MR imaging of the brain can provide substantially more information than MR imaging alone. MR imaging is also used at many centers for the detection and staging of prostate cancer, but prostate cancer is frequently not ${ }^{18} \mathrm{~F}$-FDG-avid at the time of diagnosis and ${ }^{18} \mathrm{~F}-\mathrm{FDG}$ uptake at the time of recurrence is variable $(13,14)$. Another common application of MR imaging is the imaging of primary liver tumors. However, the variable metabolic activity of these tumors has limited the clinical use of ${ }^{18}$ F-FDG PET (15). In pancreatic cancer, another common indication for oncologic MR imaging, ${ }^{18}$ F-FDG PET is limited by false-positive findings due to pancreatitis as well as by low ${ }^{18} \mathrm{~F}-\mathrm{FDG}$ uptake in tumors with substantial desmoplastic reactions (16). Because of these limitations of ${ }^{18} \mathrm{~F}$-FDG PET, demonstrating significant improvements in diagnostic accuracy and patient management with ${ }^{18}$ F-FDG PET/MR imaging compared with MR imaging alone will be challenging.

Fortunately, several promising radiopharmaceuticals in clinical development may change this situation. For example, ligands for prostate-specific membrane antigen and gastrin-releasing peptide receptors were recently demonstrated by several investigators to provide high-contrast images of primary and metastatic prostate cancers in patients (17-21). Combined with multiparametric MR imaging, these new imaging probes may be used to differentiate prostate cancer from benign diseases of the prostate, to characterize the aggressiveness of primary prostate cancer, and to localize recurrent disease in patients with biochemical recurrence. PET/MR imaging may also be used to guide radionuclide therapy of metastatic prostate cancer as a companion diagnostic technique (22). Because the high soft-tissue contrast of MR imaging is critical for the detection of primary tumors, local recurrences, and osseous metastases of prostate cancer, PET/MR imaging likely is superior to PET/CT for these applications. Retrospective fusion of independently acquired PET and MR imaging studies is principally feasible for tumors located in the pelvis, but the accuracy of coregistration is expected to be limited by the varying filling states of the bladder and rectum.

Another potential application of PET/MR imaging is the imaging of brain tumors with radiolabeled amino acids. Many studies have shown that amino acid PET provides information complementary to that provided by MR imaging $(23,24)$. Examples include better definition of the infiltration of normal brain by gliomas (25), differentiation of recurrent disease from treatment-related changes (26), assessment of the tumor response to therapy (27), and prediction of the prognosis for patients with low-grade tumors $(28,29)$. Although PET and MR imaging studies of the brain can easily be fused retrospectively, the simultaneous acquisition of PET and MR imaging studies will further improve the coregistration of PET and MR imaging information. Moreover, the pharmacokinetics of radiolabeled amino acids may allow for a dynamic PET study to be performed within the time frame of a typical MR imaging study $(28,29)$, thereby providing comprehensive characterization of brain tumors without the need for additional imaging time.

\section{NEW REGULATORY ENVIRONMENT}

In many countries, there are serious concerns about rising health care costs for aging populations (30). Although imaging tests contribute only a small portion to overall health care costs (31), they have nevertheless become a focus of efforts to curb health care costs because imaging costs have been growing at a higher rate than overall health care costs in recent years (32). Therefore, obtaining regulatory approval and reimbursement for new imaging tests and radiopharmaceuticals is considerably more challenging now than it was in the 1980s and 1990s. Regulatory agencies often require not only demonstration of increased diagnostic accuracy but also evidence for changes in patient management and improvement in patient-relevant outcomes before granting reimbursement for new imaging tests (33). As a consequence, decisions about reimbursement for imaging tests are increasingly based on the principles established 
for therapeutic agents (33). This situation represents a significant challenge for the entire field of medical imaging because the result of a diagnostic test often is only indirectly linked to patient outcome. For example, many clinicians probably consider a $30 \%$ increase in the diagnostic accuracy of cancer staging by a new imaging test to be promising. If it is (optimistically) assumed that this change in diagnostic accuracy will lead to treatment changes in $50 \%$ of patients and that these treatment changes will improve outcome in $30 \%$ of patients, then the novel imaging test will improve overall outcome in only $4.5 \%$ of studied patients $(30 \% \times 50 \% \times 30 \%)$. Demonstrating this kind of benefit in a clinical trial typically will require a randomization of several hundreds of patients $(34,35)$. Importantly, such a trial will demonstrate benefit only for a specific clinical setting, such as preoperative staging of stage II breast cancer followed by surgery.

These considerations suggest that obtaining regulatory approval for ${ }^{18} \mathrm{~F}$-FDG PET/MR imaging in indications for which ${ }^{18} \mathrm{~F}-\mathrm{FDG}$ PET/CT is already established will be challenging: because ${ }^{18} \mathrm{~F}-\mathrm{FDG}$ PET/CT is quite accurate, demonstrating improved patient outcomes will require large randomized trials, and it not clear who would fund such trials. The use of PET/MR imaging as a substitute for PET/CT in children might be an exception. Because children are more sensitive to the effects of ionizing radiation, a reduction of radiation exposure might be considered a sufficient reason for reimbursement for PET/MR imaging, provided that its diagnostic accuracy is not inferior to that of PET/CT.

\section{CONCLUSION}

PET/MR imaging is a fascinating new technology with many potential applications in oncology. The feasibility of integrated PET/MR imaging has been demonstrated by a series of clinical studies comparing ${ }^{18} \mathrm{~F}$-FDG PET/CT with ${ }^{18} \mathrm{~F}$-FDG PET/MR imaging. However, in the current regulatory environment, establishing whole-body ${ }^{18} \mathrm{~F}$ FDG PET/MR imaging as an alternative to ${ }^{18} \mathrm{~F}-\mathrm{FDG}$ PET/CT in adult patients will be challenging. Studying the use of PET/MR imaging with novel radiopharmaceuticals as an alternative to MR imaging in disease conditions for which the accuracy of ${ }^{18} \mathrm{~F}-\mathrm{FDG}$ PET and CT is limited appears to be a more promising alternative.

\section{DISCLOSURE}

No potential conflict of interest relevant to this article was reported.

\section{REFERENCES}

1. Beyer T, Townsend DW, Brun T, et al. A combined PET/CT scanner for clinical oncology. J Nucl Med. 2000;41:1369-1379.

2. Goerres GW, von Schulthess GK, Steinert HC. Why most PET of lung and headand-neck cancer will be PET/CT. $J$ Nucl Med. 2004;45(suppl 1):66S-71S.

3. Wahl RL. Why nearly all PET of abdominal and pelvic cancers will be performed as PET/CT. J Nucl Med. 2004;45(suppl 1):82S-95S.

4. Czernin J, Schelbert HR. PET/CT in cancer patient management: introduction. J Nucl Med. 2007;48(suppl 1):2S-3S.

5. Czernin J, Allen-Auerbach M, Schelbert HR. Improvements in cancer staging with PET/CT: literature-based evidence as of September 2006. J Nucl Med. 2007;48(suppl 1):78S-88S

6. Schlemmer HP, Pichler BJ, Schmand M, et al. Simultaneous MR/PET imaging of the human brain: feasibility study. Radiology. 2008;248:1028-1035.

7. Ratib O, Beyer T. Whole-body hybrid PET/MRI: ready for clinical use? Eur J Nucl Med Mol Imaging. 2011;38:992-995.

8. Bailey DL, Barthel H, Beuthin-Baumann B, et al. Combined PET/MR: where are we now? Summary Report of the Second International Workshop on PET/MR Imaging April 8-12, 2013, Tubingen, Germany. Mol Imaging Biol. March 26, 2014 [Epub ahead of print].

9. Czernin J, Ta L, Herrmann K. Does PET/MR imaging improve cancer assessments? Literature evidence from more than 900 patients. J Nucl Med. 2014; 55(suppl 2):59S-62S.
10. Huang BY, Solle M, Weissler MC. Larynx: anatomic imaging for diagnosis and management. Otolaryngol Clin North Am. 2012;45:1325-1361.

11. Kuno H, Onaya H, Fujii S, Ojiri H, Otani K, Satake M. Primary staging of laryngeal and hypopharyngeal cancer: CT, MR imaging and dual-energy CT. Eur J Radiol. 2014;83:e23-e35.

12. Gross MW, Weber WA, Feldmann HJ, Bartenstein P, Schwaiger M, Molls M. The value of F-18-fluorodeoxyglucose PET for the 3-D radiation treatment planning of malignant gliomas. Int J Radiat Oncol Biol Phys. 1998;41:989-995.

13. Jadvar H, Desai B, Ji L, et al. Baseline ${ }^{18} \mathrm{~F}-\mathrm{FDG}$ PET/CT parameters as imaging biomarkers of overall survival in castrate-resistant metastatic prostate cancer. J Nucl Med. 2013;54:1195-1201.

14. Meirelles GS, Schoder H, Ravizzini GC, et al. Prognostic value of baseline $\left[{ }^{18} \mathrm{~F}\right]$ fluorodeoxyglucose positron emission tomography and ${ }^{99 \mathrm{~m}} \mathrm{Tc}-\mathrm{MDP}$ bone scan in progressing metastatic prostate cancer. Clin Cancer Res. 2010;16:6093-6099.

15. Talbot JN, Fartoux L, Balogova S, et al. Detection of hepatocellular carcinoma with PET/CT: a prospective comparison of ${ }^{18} \mathrm{~F}$-fluorocholine and ${ }^{18} \mathrm{~F}-\mathrm{FDG}$ in patients with cirrhosis or chronic liver disease. J Nucl Med. 2010;51:1699-1706.

16. Sendler A, Avril N, Helmberger H, et al. Preoperative evaluation of pancreatic masses with positron emission tomography using ${ }^{18} \mathrm{~F}$-fluorodeoxyglucose: diagnostic limitations. World J Surg. 2000;24:1121-1129.

17. Afshar-Oromieh A, Malcher A, Eder M, et al. PET imaging with a $\left[{ }^{68} \mathrm{Ga}\right]$ galliumlabelled PSMA ligand for the diagnosis of prostate cancer: biodistribution in humans and first evaluation of tumour lesions. Eur J Nucl Med Mol Imaging. 2013;40:486-495.

18. Cho SY, Gage KL, Mease RC, et al. Biodistribution, tumor detection, and radiation dosimetry of ${ }^{18} \mathrm{~F}-\mathrm{DCFBC}$, a low-molecular-weight inhibitor of prostatespecific membrane antigen, in patients with metastatic prostate cancer. $\mathrm{J} \mathrm{Nucl}$ Med. 2012;53:1883-1891.

19. Kähkönen E, Jambor I, Kemppainen J, et al. In vivo imaging of prostate cancer using $\left[{ }^{68} \mathrm{Ga}\right]-$ labeled bombesin analog BAY86-7548. Clin Cancer Res. 2013;19:5434-5443.

20. Wieser G, Mansi R, Grosu AL, et al. Positron emission tomography (PET) imaging of prostate cancer with a gastrin releasing peptide receptor antagonist: from mice to men. Theranostics. 2014;4:412-419.

21. Afshar-Oromieh A, Haberkorn U, Schlemmer HP, et al. Comparison of PET/CT and PET/MRI hybrid systems using a ${ }^{68} \mathrm{Ga}$-labelled PSMA ligand for the diagnosis of recurrent prostate cancer: initial experience. Eur J Nucl Med Mol Imaging. 2014;41:887-897.

22. Zechmann CM, Afshar-Oromieh A, Armor T, et al. Radiation dosimetry and first therapy results with a ${ }^{124} \mathrm{I} /{ }^{131} \mathrm{I}$-labeled small molecule (MIP-1095) targeting PSMA for prostate cancer therapy. Eur J Nucl Med Mol Imaging. February 28, 2014 [Epub ahead of print].

23. Filss CP, Galldiks N, Stoffels G, et al. Comparison of ${ }^{18}$ F-FET PET and perfusion-weighted MR imaging: a PET/MR imaging hybrid study in patients with brain tumors. J Nucl Med. 2014;55:540-545.

24. Rahm V, Boxheimer L, Bruehlmeier M, et al. Focal changes in diffusivity on apparent diffusion coefficient MR imaging and amino acid uptake on PET do not colocalize in nonenhancing low-grade gliomas. J Nucl Med. 2014;55:546-550.

25. Grosu AL, Weber WA, Riedel E, et al. L-(methyl- $\left.{ }^{11} \mathrm{C}\right)$ methionine positron emission tomography for target delineation in resected high-grade gliomas before radiotherapy. Int J Radiat Oncol Biol Phys. 2005;63:64-74.

26. Grosu AL, Astner ST, Riedel E, et al. An interindividual comparison of $O-\left(2-\left[{ }^{18} \mathrm{~F}\right]\right.$ fluoroethyl)-L-tyrosine (FET)- and L-[methyl- $\left.{ }^{11} \mathrm{C}\right]$ methionine (MET)-PET in patients with brain gliomas and metastases. Int J Radiat Oncol Biol Phys. 2011;81:1049-1058.

27. Schwarzenberg J, Czernin J, Cloughesy TF, et al. Treatment response evaluation using ${ }^{18} \mathrm{~F}$-FDOPA PET in patients with recurrent malignant glioma on bevacizumab therapy. Clin Cancer Res. March 31, 2014 [Epub ahead of print].

28. Pöpperl G, Kreth FW, Mehrkens JH, et al. FET PET for the evaluation of untreated gliomas: correlation of FET uptake and uptake kinetics with tumour grading. Eur J Nucl Med Mol Imaging. 2007;34:1933-1942.

29. Galldiks N, Stoffels G, Ruge MI, et al. Role of $O-\left(2-{ }^{18} \mathrm{~F}\right.$-fluoroethyl $)$-L-tyrosine PET as a diagnostic tool for detection of malignant progression in patients with low-grade glioma. J Nucl Med. 2013;54:2046-2054.

30. Sullivan R, Peppercorn J, Sikora K, et al. Delivering affordable cancer care in high-income countries. Lancet Oncol. 2011;12:933-980.

31. Yang Y, Czernin J. Contribution of imaging to cancer care costs. J Nucl Med. 2011;52(suppl 2):86S-92S.

32. Iglehart JK. Health insurers and medical-imaging policy: a work in progress. N Engl J Med. 2009;360:1030-1037.

33. Ware RE, Hicks RJ. Doing more harm than good? Do systematic reviews of PET by health technology assessment agencies provide an appraisal of the evidence that is closer to the truth than the primary data supporting its use? J Nucl Med. 2011;52(suppl 2):64S-73S.

34. Vach W, Hoilund-Carlsen PF, Gerke O, Weber WA. Generating evidence for clinical benefit of PET/CT in diagnosing cancer patients. J Nucl Med. 2011; 52(suppl 2):77S-85S.

35. Weber WA. Is there evidence for evidence-based medical imaging? J Nucl Med. 2011;52(suppl 2):74S-76S. 